\title{
Morphological applicability of currently available stent grafts in the endovascular repair of asymptomatic abdominal aortic aneurysm in East-Central European patients
}

\author{
Jolanta Tomczak ${ }^{1}$, Łukasz Dzieciuchowicz² \\ ${ }^{1}$ Department of Vascular and Endovascular Surgery, Angiology and Phlebology, Poznan University of Medical Sciences, Poznan, Poland \\ ²Department of Vascular Surgery and Vascular Diseases, Institute of Medical Sciences, University of Zielona Góra, Zielona Góra, Poland
}

Adv Interv Cardiol 2021; 17, 1 (63): 93-100

DOI: https://doi.org/10.5114/aic.2021.104774

\begin{abstract}
A bstract
Introduction: Currently, there is a wide range of commercially available devices for endovascular aneurysm repair (EVAR) that differ in terms of both anatomical requirements and the technology and technique of deployment.

Aim: To assess the applicability of currently commercially available devices for EVAR in the treatment of an asymptomatic abdominal aneurysm (AAA).

Material and methods: The study group included 100 patients with infrarenal AAA with a maximum diameter $\geq 50 \mathrm{~mm}$, qualified for invasive treatment at the University Hospital in 2013-2014. The aortoiliac morphological characteristics of the AAA were evaluated on preoperative computed tomography angiograms using the OsiriX DICOM viewer in the 3D-MPR mode. The morphological applicability of 14 types of CE-marked and FDA-approved stent grafts was determined based on their instructions for use (IFU).

Results: EVAR was feasible with at least one of the analysed devices in $68 \%$ of patients. The morphological applicability was as follows: Excluder Conformable (65\%), Ovation iX (51\%), Endurant II (47\%), Treo (45\%), Excluder C3 (45\%), AFX 2 (45\%), Incraft (44\%), E-tegra (44\%), Zenith Alfa (41\%), Zenith Flex (40\%), Anaconda (39\%) Aorfix (37\%), Altura (34\%), and E-vita (20\%). The differences in the stent graft applicability were statistically significant $(p<0.001)$. A wide diameter of the common iliac artery, angulated proximal neck, and diameter of proximal neck out of range constituted the most frequent causes of EVAR inapplicability.

Conclusions: The IFU-based applicability of currently available AAA stent graft systems differs significantly. Despite the constant evolution of EVAR technology, at least $32 \%$ of AAA will require a different therapeutic approach.
\end{abstract}

Key words: infrarenal abdominal aortic aneurysms, endovascular aneurysm repair, stent graft, instructions for use.

S u m m a ry

The results of IFU-based feasibility of modern endovascular aneurysm repair (EVAR) in a range of 100 patients with asymptomatic abdominal aortic aneurysm (AAA) in the East-Central European population are presented. The overall EVAR feasibility reached $68 \%$, with individual stent graft system applicability ranging from $20 \%$ to $65 \%$. The most frequent causes of EVAR inapplicability were the wide diameter of the common iliac artery, its angulated proximal neck, and the diameter of its proximal neck out of range. Excluder Conformable seems to be the most applicable stent graft system. One-third of asymptomatic AAAs require an open repair or more advanced EVAR technology.

\section{Introduction}

Continuously increasing applicability of endovascular aneurysm repair (EVAR) due to the development of new devices and techniques of deployment and the evolution of manufacturers' instructions for use (IFU) offer a pos- sibility to treat abdominal aortic aneurysms (AAA) with complex anatomies. As a result, there are several commercially available stent graft systems that have different criteria for use, and also differ in regard to technical aspects of implantation. Since the clinical success of EVAR

\section{Corresponding author:}

Jolanta Tomczak MSc, Department of Vascular and Endovascular Surgery, Angiology and Phlebology, Poznan University of Medical Sciences, Poznan, Poland, phone: +48 6185491 41, e-mail: jolantatomczak.pte@gmail.com

Received: 20.11.2020, accepted: 16.12 .2020$. 
depends on proper patient and stent graft selection and expeditious stent graft implantation, it can be assumed that one has to limit their stent graft armamentarium to a minimum number of device types to gain sufficient proficiency in EVAR planning and performance. It is very important to apply EVAR within IFU also with regard to medico-legal issues. Though some EVAR feasibility studies have been published, they did not concern East-Central European populations, and some of them were based on the old generation of stent grafts, which are no longer in use and had a retrospective nature [1-6].

\section{Aim}

The aim of this study was to determine the applicability of currently registered and commercially available stent graft systems for endovascular treatment of AAAs in East-Central European populations.

\section{Material and methods}

\section{Patients}

Computed tomography angiograms (CTAs) of 100 consecutive eligible patients referred for the elective repair of an infrarenal AAA to the University Hospital's Department of Vascular Surgery in the years 2013-2014 were analysed. The study complies with the Declaration of Helsinki, and ethical approval for this study was waived by the institutional bioethical committee because of the absence of signs of a medical experiment. All patients admitted to the hospital with a diagnosis of an infrarenal AAA were pre-screened. The inclusion criteria were the presence of an asymptomatic AAA of the maximum diameter $\geq 50 \mathrm{~mm}$ and good quality CTAs with a slice thickness of at least $0.625 \mathrm{~mm}$ comprising the entire abdominal aorta and the iliac and common femoral arteries. Patients with para- and suprarenal aneurysms, dissections, penetrating aortic ulcers, mycotic aneurysms, and previous aortoiliac interventions, with either suspected or diagnosed connective tissue disorders, were excluded from the study. Case enrolment was closed after recruiting 100 patients.

The suitability for the EVAR was specified based on a review of their CTA scans before the patients were qualified for either EVAR or an open surgical repair. The data on patients' demographics and cardiovascular risk factors were collected.

\section{Measures and definitions}

The morphological analysis of anonymised CTAs was performed using the OsiriX DICOM (Digital Image and Communication in Medicine) viewer (Pixmeo Sàrl, Bernex, Switzerland) in the 3D multiplanar reconstruction mode. Diameter measurements were made orthogonally to the centreline of the artery from the outer wall to the outer wall for the outer diameter and from the inner wall to the inner wall for the inner diameter. The length measurements were made along the centreline. The proximal neck was defined as a segment of the non-aneurysmal aorta between the lower renal artery and the beginning of the aneurysm. The following morphological parameters were assessed: a) diameter, length, $\alpha$ and $\beta$ angulation as well as presence and extent of thrombus and calcification of the proximal neck, b) diameter of the distal neck, c) maximum aneurysm length, and d) diameters and anatomy of the common (CIA), external (EIA), and internal iliac arteries (IIA). Factors such as the shape of the neck (conical) and iliac artery tortuosity were not taken into consideration because they were not mentioned in the IFU.

The feasibility assessment for EVAR was determined based on the latest available IFU of the analysed stent grafts (Table I), which were either FDA-approved or had a CE mark. Fourteen EVAR systems were analysed: AFX 2 Endovascular AAA System (Endologix, Irvine, CA), Altura Endograft System and Aorfix (Lombard Medical, Oxfordshire, United Kingdom), Anaconda (Vascutek, Inchinnan, United Kingdom), Endurant II Stent Graft System (Medtronic Cardiovascular, Santa Rosa, CA, USA), Excluder C3 and Excluder Conformable stent graft (W.L. Gore and Associates, Flagstaff, AZ, USA), E-vita and E-tegra abdominal stent graft (Jotec, Hechingen, Germany), Incraft stent graft system (Cordis Corporation, Bridgewater, NJ, USA), Ovation iX (Endologix, Santa Rosa, CA, USA), Treo Abdominal stent graft system (Bolton Medical Inc., Sunrise, FL, USA), Zenith Alfa and Zenith Flex (Cook Medical, Bloomington, IN, USA).

An AAA was considered suitable for EVAR with a particular stent graft system when the morphological characteristics fulfilled all the anatomical recommendations of the IFU. The IFU criteria of the analysed stent graft systems are presented in Table I.

Assuming that pooling two EVAR systems will increase the applicability of EVAR, all the analysed devices were combined in pairs, and the feasibility for EVAR for each pair was calculated.

\section{Statistical analysis}

The statistical analysis was performed with the GraphPad Prism software (version 6.04). Qualitative parameters were expressed as percentages, while continuous variables were reported as a median and quartiles. Qualitative parameters were compared using the $\chi^{2}$ test. The observed differences were considered statistically significant at $p<0.05$.

\section{Results}

\section{Characteristics of patients}

Most of the study patients were male (82\%), with a median of age of 69.5 (63-75) years. The median maximum diameter of AAA was $61.0(55.0-69.5) \mathrm{mm}$ and did 


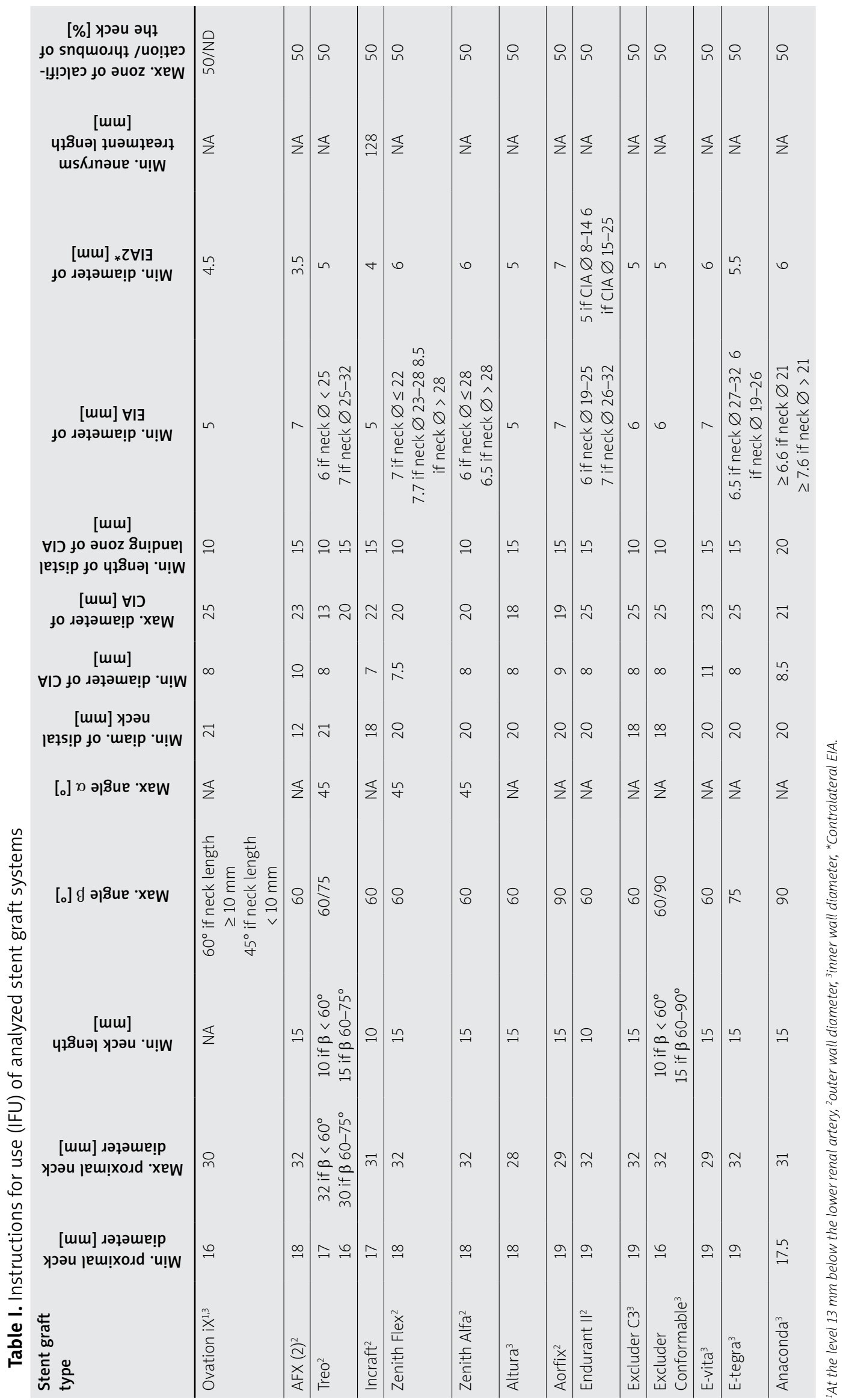


not differ between men and women, 61.0 (55.0-69.0) $\mathrm{mm}$ and $62.5(53.0-70.0) \mathrm{mm}$ respectively ( $p=0.656)$. Sixty-five percent of patients had hypertension, $44 \%$ had coronary artery disease, 34\% had hyperlipidaemia, 25\% had peripheral artery disease, $23 \%$ had diabetes, $7 \%$ had renal insufficiency (7\%), $24 \%$ were obese $\left(\mathrm{BMI}>30 \mathrm{~kg} / \mathrm{m}^{2}\right)$, and $20 \%$ were active smokers.

\section{Morphological criteria for EVAR}

Endovascular repair of the abdominal aortic aneurysm would be feasible in $68 \%$ of the study patients if the IFU were rigorously followed, and $32 \%$ were not suitable for any of the analysed stent grafts.

The largest number of patients, as many as $65 \%$, were morphologically suitable for the Excluder Conformable stent graft, followed by Ovation iX and Endurant II, with the applicability rate of $51 \%$ and $47 \%$, respectively. The most unsuitable stent grafts for the analysed group of patients were E-vita and Altura, with suitability of $20 \%$ and $34 \%$, respectively (Figure 1). The differences in stent graft applicability were statistically significant $(p<0.001)$. The main limiting criteria within the analysed stent graft systems in order of prevalence are presented in Table II. Three patients demonstrated anatomic characteristics that made them suitable candidates for implantation of only one device: Excluder Conformable (2) and AFX (1).

The maximal diameter of the CIA was the most prevalent cause of EVAR unsuitability that occurred depending on the stent graft system in $18-63 \%$ of patients, with Excluder and Ovation systems being the most and the E-vita system being the least applicable for wide CIAs. The CIA diameter was also the most common as a single limiting factor, which occurred in $9-28 \%$ of patients in 13 stent grafts. The proximal neck diameter out of range was the second most frequent cause of unsuitability, which occurred depending on the stent graft system in

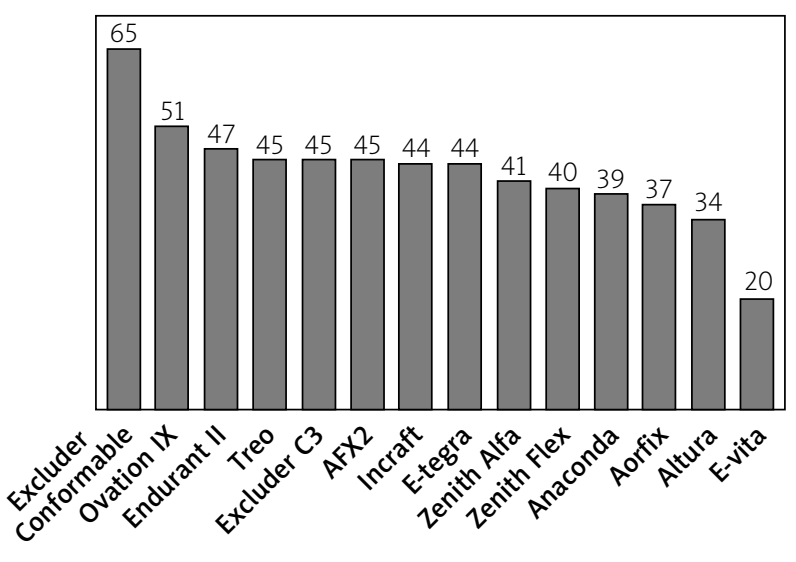

Figure 1. Percentage of patients fitting the Instructions for use anatomical criteria of applicability of analyzed stent-graft systems
5-27\% of patients, with the Excluder Conformable system being the most and the E-vita system being the least applicable for out of range diameter of proximal neck; however, out of range proximal neck diameter was the single cause of unsuitability only in one device. The $\beta$ angle was the third most prevalent cause of EVAR unsuitability, occurring in $6-12 \%$ of patients in 10 out of 14 evaluated stent grafts and a single cause of unsuitability in one device. Excluder Conformable, E-tegra, Anaconda, Aorfix, and Treo systems were the most applicable for a large $\beta$ angle. The presence of > $50 \%$ thrombus of the proximal neck was found in $9 \%$ of patients. A short proximal neck resulted in unsuitability in 5 to $8 \%$ of the cases for all the devices, while a distal neck diameter was a limitation in $0-8 \%$ of the cases. The problem of the diameter of external iliac arteries was found minor in terms of suitability of the studied EVAR, except for Aorfix, where $11 \%$ of cases had an unsuitable EIA diameter. Major reasons of unsuitability for the evaluated EVAR systems are shown in Table II.

The pooled applicability exceeded the highest individual suitability rate for the pairs: Excluder Conformable Treo by $2 \%$, for Excluder Conformable - AFX 2 by $1 \%$. The details are presented in Table III. Pairing Excluder Conformable with Treo made it possible to treat two more patients with a narrow proximal aortic neck and pairing the Excluder Conformable with AFX 2 made it possible to perform EVAR in one more patient with a narrow distal aortic neck.

\section{Discussion}

The present study aimed to evaluate the number of patients with asymptomatic AAAs who can be treated by EVAR with commercially available stent graft devices in conformity with the IFU. Although studies on EVAR suitability have been previously published, the present study has some unique features. A few former studies evaluated retrospectively the anatomical features in patients who had undergone endovascular repair procedures, or included only selected patients $[2,7,8]$, whereas the present study analysed all patients with a diagnosed infrarenal AAA with the maximum aneurysm sac diameter of $50 \mathrm{~mm}$ or larger, regardless of the ultimate treatment plan, which resulted in a more representative group of AAA patients. Also, to the best of our knowledge, this is the first published study on the feasibility of the applicability of fourteen FDA-approved commercial devices among the East-Central European AAA population. Moreover, due to the constant accumulation of knowledge and the development of EVAR technology, the variety of commercially available EVAR devices is evolving. Most of the studies on the subject are more than 5 years old, and over that period of time, new devices have appeared, whereas some others disappeared from the market, so there is always a need to update the information on the EVAR applicability $[1,4,7,8]$. 

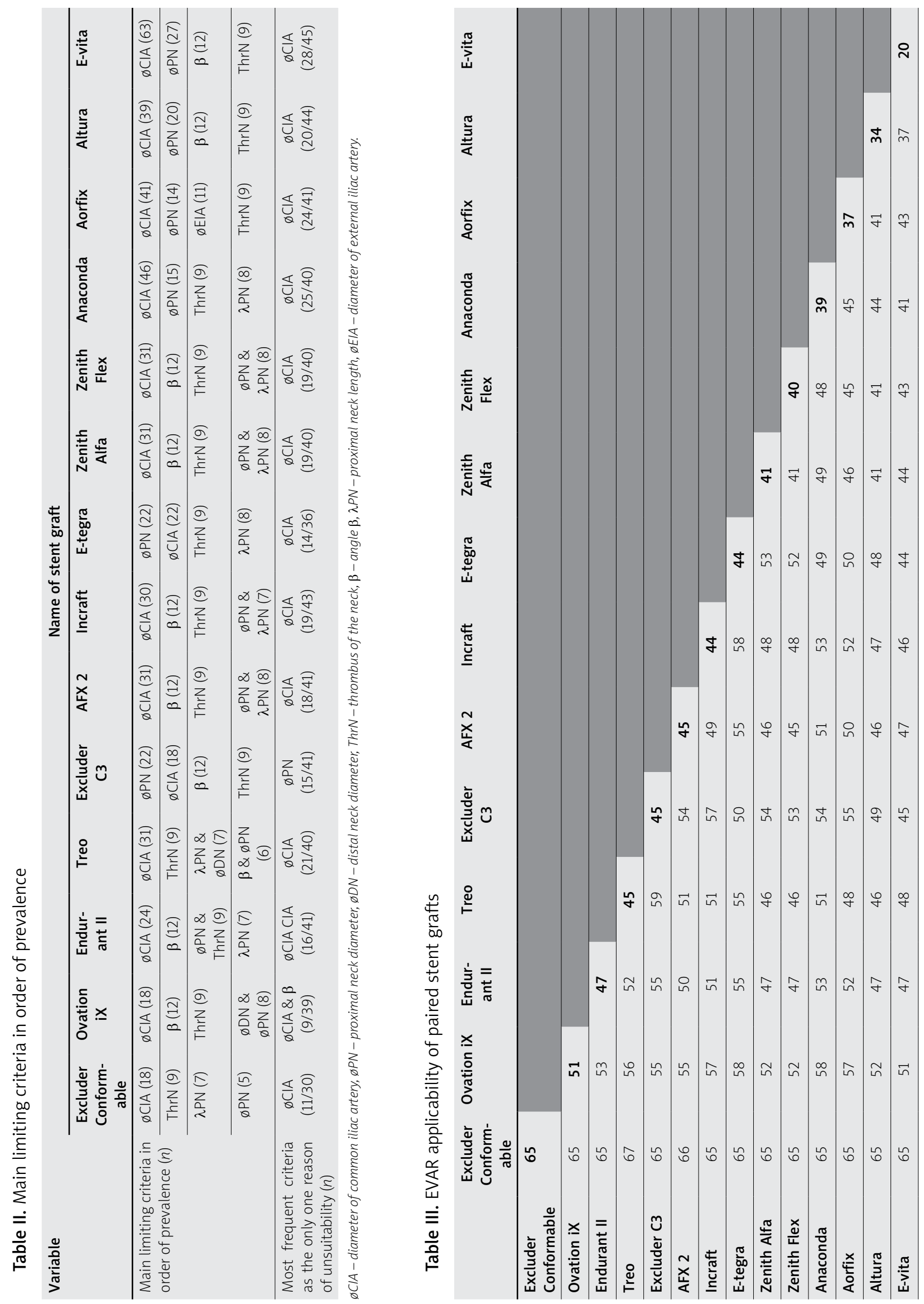
The compliance with IFU criteria not only positively influences the long-term durability of EVAR but is also important from the medico-legal and ethical standpoint $[7,9]$. The present study showed that $68 \%$ of the analysed patients met the IFU morphological criteria for at least one of the currently commercially available stent grafts. The most common barriers for using the selected stent graft were a wide diameter of the common iliac artery, a large $\beta$ angle, and an inadequate diameter of the proximal neck. Proximal neck length, the diameter of the distal neck, and the presence of a moderate or severe thrombus of the proximal neck had a smaller influence on the stent graft suitability rate. Proximal neck calcification and $\alpha$ angulation and the diameter of the access vessels appear to play a minor role in the applicability of EVAR in the studied group.

In $20 \%$ to $40 \%$ of the patients treated with EVAR, the aneurysm extends to at least one and in 11-14\% to both CIAs [3, 10, 11]. Based on the results from the current study, the diameter of the common iliac arteries constitutes a crucial criterion of whether a patient with AAA can be treated using EVAR. That is why EVAR systems such as Excluder, Endurant II, E-tegra, and Ovation iX, which within their standard configuration offer wide iliac extensions making it possible to treat patients with the CIA diameter up to $25 \mathrm{~mm}$, demonstrated higher applicability in this study. The Zenith stent graft system offers a wide iliac extension as a custom-made device. That is why it was not taken into account in this study, although it may expand its real-life applicability. It has to be remembered, however, that the risk of late type IB endoleak is several times higher in patients treated with iliac limbs $\geq 20 \mathrm{~mm}$ as compared to patients treated with a distal iliac limb diameter $<20 \mathrm{~mm}$ [12]. To solve this therapeutic problem, some manufacturers have developed elements that allow the endograft to be extended into the external iliac artery, maintaining the perfusion of the internal iliac artery by a side branch that may further extend the applicability of EVAR [11].

In a study of 235 Greek patients with AAA, higher EVAR applicability rates than the ones observed in the present study were reported. The most applicable were Endurant II, Ovation iX, and Treo devices with the suitability rate of $80.7 \%, 78.9 \%$, and $74.9 \%$, respectively. The lowest feasibility rate was observed for Incraft, Aorfix, and Altura stent grafts with the suitability rates of $48.5 \%$, $42.7 \%$, and $34.5 \%$, respectively. There were two criteria significantly limiting the suitability: the neck dimensions, and the CIA diameter. In that report, cases with extreme angulation, the presence of thrombus, or atherosclerosis of the CIAs had been excluded from their study, as they had been immediately considered unsuitable for endovascular repair [2]. Consequently, the cohort does not comprise a complete view of the variety of anatomies that would correspond to real-life clinical settings. The present work contains all infrarenal AAA patients, even those with adverse anatomies, which in the opinion of the authors gives a more reliable group representation and can partially explain the observed lower applicability rates. The differences in results of EVAR suitability may also be caused by the differences in aneurysm morphology associated with the patients' region.

The results obtained in this study do not seem to reflect the real-life situation. In the first quarter of 2019, Endurant II was the most frequently implanted stent graft, with almost $44 \%$ of market share, followed by Excluder (23\%) and Zenith (16.5\%) [13]. However, in the present study, Endurant II is surpassed by two stent grafts: Excluder Conformable and Ovation iX. There may be several reasons for this discrepancy. Endurant II, in distinction to Excluder, has a suprarenal fixation. Since it does not change the IFU and does not influence the results, the type of proximal fixation should not influence the applicability. However, in the case of a suboptimal neck, many vascular surgeons prefer to use a device with suprarenal fixation. Endurant II is a successor of the Talent stent graft - they are both produced by the same company, Medtronic, present on the EVAR market for more than 30 years, enough time to have gained considerable trust among vascular surgeons. The same is true with regards to the Zenith and Excluder grafts, and it may explain the prevalence of these three systems over other grafts.

The data about the market share of the stent graft system come from the first quarter of the last year. At that time, Excluder Conformable, which has increased the applicability of the system in comparison to Excluder C3, was not available, which may also explain the prevalence of the Endurant II system. This fact partially explains the highest applicability of Endurant II in the aforementioned study comprising 235 patients from Greece [2]. In that study, however, Endurant II was better than the Treo, Ovation iX, and E-tegra systems, which may have resulted from different patients' characteristics with regard to proximal neck morphology, more angulated necks in our patients, and shorter proximal necks in Greek patients. Furthermore, although it is not stated in the standard IFU, since 2016, Endurant II has been approved for use with chimney grafts, which may have further increased its applicability. Additionally, since September 2017, the IFU criteria of Endurant II have been extended to a short neck $\geq 4 \mathrm{~mm}$, on condition that the Heli-FX EndoAnchor System fixation is applied. Because this approach requires additional devices to be used and, in our opinion, may not be durable, this indication was not included in the analysis. The data on the long-term results of that approach are still lacking $[14,15]$. As a matter of fact, in the biggest registry reporting very good long-term results of infrarenal AAA treatment with Endurant, only $12 \%$ of patients had a proximal neck $<15 \mathrm{~mm}$ [16].

The Ovation ix grafts demonstrated a very high applicability rate both in the study from Greece and in the 
present one. Besides the possibility of treatment of patients with CIA ID up to $25 \mathrm{~mm}$, it is unique in its proximal neck criteria that require a 16 to $30 \mathrm{~mm}$ ID only at $13 \mathrm{~mm}$ below the lower renal artery without specifying what the diameter above and below that level is. The seal at this level is ensured by a polymer filled ring. So far, its limited use in real life may result from low confidence in the durability of a proximal seal, especially after the disappointing result of the endovascular aneurysm sealing technology (EVAS) [17, 18]. However, the Ovation iX stent graft, in distinction to EVAS, also has an active suprarenal fixation system. And indeed, a recently published study of 1296 patients treated with that device, $50 \%$ of whom had complex aortic anatomies, demonstrated freedom from type IA endoleak in 95.8\% and freedom from device-related reintervention of $92.4 \%$ over a period of 5 years [19]. A probable introduction of the Ovation Alto device in the near future with the sealing ring to be placed only $7 \mathrm{~mm}$ below the lower renal artery may further increase the applicability rate of this device [20].

It has to be noted, however, that the IFU criteria of currently commercially available stent grafts extend beyond what is considered the optimal anatomy for EVAR. On the one hand, it reflects the pursuit of the industry to cover a wide range of anatomies of AAAs. But on the other hand, one has to bear in mind that widening of anatomic criteria may compromise long-term results of EVAR. In a study that analysed the data of a large, multicentre cohort of 10,228 patients treated with EVAR, the independent morphological predictors of the AAA sac enlargement included an aortic neck diameter $\geq 28 \mathrm{~mm}$, an aortic neck angle $>60^{\circ}$, and a common iliac artery diameter $>20 \mathrm{~mm}$ [9].

A bitter lesson was learned from the experience with Endovascular Aneurysm Sealing System (EVAS). According to its primary IFU, based on the analysis of 776 patients with $A A A$, more than $70 \%$ were considered suitable for EVAS [18]. Despite encouraging early reports, the device has been recalled due to poor long-term results, associated with type IA endoleak, graft migration, sac expansion, and the occurrence of an aorto-duodenal fistula [17, 18].

Pooling the morphological criteria of the most applicable device with any other only slightly increased the applicability of EVAR. So, it seems advisable that centres treating infrarenal AAAs should limit their armamentarium to two or three systems in order to gain maximum knowledge and expertise in device sizing, implantation techniques, and results without compromising the overall applicability of EVAR.

\section{Conclusions}

Within the East-Central European AAA population, the suitability rates for the analysed devices vary widely from $65 \%$ up to $20 \%$. CIA diameters, as well as out of range and angulated proximal neck, are the morphological parameters significantly influencing the suitability rate of the evaluated stent grafts. A combination of the most applicable stent graft with any other device increases the applicability of EVAR only slightly. Thus, more advanced endovascular technologies, as well as open surgical repair techniques, are required to be able to treat all ranges of the anatomy of infrarenal AAA.

\section{Conflict of interest}

The authors declare no conflict of interest.

\section{References}

1. Park KH, Lim C, Lee JH, et al. Suitability of endovascular repair with current stent grafts for abdominal aortic aneurysm in Korean patients. J Korean Med Sci 2011; 26: 1047-51.

2. Patelis ND, Malli A, Mylonas KS, et al. Suitability study of current endovascular aortic repair devices based on real-life anatomic data. Expert Rev Med Devices 2019; 16: 165-71.

3. Dzieciuchowicz $Ł$, Tomczak J, Strauss E, et al. Morphology-related limitations of EVAR applicability in the treatment of AAA in West-Central Poland. Ann Vasc Surg 2018; 52: 49-56.

4. Carpenter JP, Baum RA, Barker CF, et al. Impact of exclusion criteria on patient selection for endovascular abdominal aortic aneurysm repair. J Vasc Surg 2001; 34: 1050-4.

5. Zarins CK, Wolf YG, Lee WA, et al. Will endovascular repair replace open surgery for abdominal aortic aneurysm repair? Ann Surg 2000; 232: 501-7.

6. Treiman GS, Lawrence PF, Edwards WH Jr, et al. An assessment of the current applicability of the EVT endovascular graft for treatment of patients with an infrarenal abdominal aortic aneurysm. J Vasc Surg 1999; 30: 68-75.

7. Abbruzzese TA, Kwolek CJ, Brewster DC, et al. Outcomes following endovascular abdominal aortic aneurysm repair (EVAR): an anatomic and device-specific analysis. J Vasc Surg 2008; 48: 19-28.

8. Walker J, Tucker LY, Goodney P, et al. Adherence to endovascular aortic aneurysm repair device instructions for use guidelines has no impact on outcomes. J Vasc Surg 2015; 61: 1151-9.

9. Schanzer A, Greenberg RK, Hevelone N, et al. Predictors of abdominal aortic aneurysm sac enlargement after endovascular repair. Circulation 2011; 123: 2848-55.

10. Hobo R, Sybrandy JE, Harris PL, et al. Endovascular repair of abdominal aortic aneurysms with concomitant common iliac artery aneurysm: outcome analysis of the EUROSTAR experience. J Endovasc Ther 2008; 15: 12-22.

11. Marques de Marino P, Botos B, Kouvelos G, et al. Use of bilateral cook zenith iliac branch devices to preserve internal iliac artery flow during endovascular aneurysm repair. Eur J Vasc Endovasc Surg 2019; 57: 213-9.

12. Gray D, Shahverdyan R, Reifferscheid V, et al. EVAR with flared iliac limbs has a high risk of late type $1 \mathrm{~b}$ endoleak. Eur J Vasc Endovasc Surg 2017; 54: 170-6.

13. https://www.bibamedtech.com/biba-briefings-top-stories/aortic-insights-q1-2019/ [accessed 28 May 2020].

14. Arko FR $3^{\text {rd }}$, Stanley GA, Pearce BJ, et al. Endosuture aneurysm repair in patients treated with Endurant II/IIs in conjunction with Heli-FX EndoAnchor implants for short-neck abdominal aortic aneurysm. J Vasc Surg 2019; 70: 732-40. 
15. Qamhawi Z, Barge TF, Makris GC, et al. Editor's choice - systematic review of the use of endoanchors in endovascular aortic aneurysm repair. Eur J Vasc Endovasc Surg 2020; 59: 748-56.

16. Teijink JAW, Power AH, Böckler D, et al. Editor's choice - five year outcomes of the endurant stent graft for endovascular abdominal aortic aneurysm repair in the ENGAGE Registry. Eur J Vasc Endovasc Surg 2019; 58: 175-81.

17. Karthikesalingam A, Cobb RJ, Khoury A, et al. The morphological applicability of a novel endovascular aneurysm sealing (EVAS) system (Nellix) in patients with abdominal aortic aneurysms. Eur J Vasc Endovasc Surg 2013; 46: 440-5.

18. Stenson KM, de Bruin JL, Loftus IM, et al. Migration and sac expansion as modes of midterm therapeutic failure after endovascular aneurysm sealing: 295 cases at a single center. J Vasc Surg 2019: S0741-5214; 31312-6.

19. Swerdlow NJ, Lyden SP, Verhagen HJM, et al. Five-year results of endovascular abdominal aortic aneurysm repair with the Ovation abdominal stent graft. J Vasc Surg 2020; 71: 1528-37.

20. Holden A, Lyden S. Initial experience with polymer endovascular aneurysm repair using the Alto stent graft. J Vasc Surg Cases Innov Tech 2020; 6: 6-11. 